\title{
AGRICULTURA URBANA PARA LA SOSTENIBILIDAD DE LAS CIUDADES
}

Fabiola Alvino

\section{Resumen}

Reflexiones sobre la Agricultura Urbana teniendo en cuenta los aspectos sociales, económicos y ambientales que inciden sobre su desarrollo y aplicación, bajo la concepción del desarrollo sostenible. Los temas involucrados se refieren a la insuficiencia alimentaria, la agricultura ecológica, la educación medioambiental, la calidad de vida, la degradación ambiental, entre otros. Entre los principales instrumentos considerados en la contribución de la agricultura urbana a la sostenibilidad de las ciudades, se cuentan: el empleo que generan, la reducción de índices de contaminación atmosférica, la protección de fuentes de agua, la recreación social.

Palabras clave: agricultura urbana, agricultura periurbana, huertos urbanos, agricultura ecológica, seguridad alimentaria, participación ciudadana, educación medioambiental, área verde productiva, sostenibilidad.

\section{Abstract}

Reflections about the Urban Agriculture considering social, economic and environmental aspects which affect their development and implementation, with the approach of the sustainable development. The involved subjects talk about the insufficiency nourishing, ecological agriculture, environmental education, quality of life, environmental degradation, among others. Between the main instruments considered in the contribution of urban agriculture to the sustainability of the cities, we have: the use that generates the reduction of levels of atmospheric pollution, the protection of water sources, and social recreation.

Key words: urban agriculture, peri-urban agriculture, urban orchards, ecological agriculture, food safety, citizen participation, environmental education, productive green area, sustainability. 


\section{LA CIUDAD SOSTENIBLE}

El concepto de sostenibilidad, que adquiere carta de ciudadanía universal en 1987, con el Informe Brundtland, "Nuestro futuro común", producto del trabajo de la Comisión Mundial sobre Medio Ambiente y Desarrollo (CMMAD) organizada por la ONU, desde entonces se ha venido ramificando en diversos campos del pensamiento y de la actividad social contemporánea. Una de las ramas más vigorosas es la que asocia sostenibilidad con desarrollo, concebidas como interdependientes e interinfluyentes. De alli que el deterioro ambiental se derive de los obstáculos que éste pueda representar para el desarrollo; es pues una perspectiva antropocentrista, distinta a la ecocentrista que primaba en la década del setenta (Pierri, 2005).

Posteriormente a la Cumbre de la Tierra o Cumbre Río, de 1992, un capítulo particularmente importante en la concepción de la sostenibilidad es el enfoque de las ciudades, en la medida que más de la mitad de la humanidad vive en centros urbanos. Así, en el capítulo 7 de la Agenda 21 trata, junto a otros aspectos concurrentes, del tema del deterioro de los asentamientos humanos, y se señala que los objetivos del tratamiento de los asentamientos humanos es mejorar la calidad social, económica y ambiental de la vida urbana y optimizar las condiciones de vida y de trabajo de todos.

En 1996selleva a cabo, en Estambul, la Conferencia de las Naciones Unidas sobre los Asentamientos Humanos, denominada Hábitat II. En este evento se estableció que "El Programa de Hábitat, tiene por objetivo ser una movilización mundial de acción a todos los niveles con objeto de lograr el desarrollo sostenible de todas las ciudades, pueblos y aldeas del mundo durante las dos primeras décadas del próximo siglo". El programa parte del principio de que toda población requiere una vivienda adecuada $\mathrm{y}$ servicios básicos, un medio ambiente sano y seguro, y el empleo productivo elegido libremente.

En la búsqueda de Ciudades Sostenibles, el territorio se integra al balance y al equilibrio de los asuntos humanos y estos a su vez buscan no transgredir los limites de la sustentabilidad que ofrece el territorio buscando una armonía simbiótica.

En investigaciones recientes, se señalan tres condiciones que deben estar presentes en una ciudad para alcanzar la sostenibilidad:

- Asignación de "los usos de suelo en forma mixta, para evitar los grandes desplazamientos en el espacio".

- Poseer "una forma compacta, con el fin de reducir distancias en los desplazamientos y con ello evitar el alto consumo de combustible".

- Presentar "bajos niveles de pobreza, una base económica diversificada, y conservación de las áreas verdes, sin basarse en el uso del automóvil privado".

La ciudad sostenible no es solo una solución urbanística al problema de 
la ocupación de un territorio; es una visión dinámica e interrelacionada que busca una relación directa entre las estructuras fisicas que la conforman con el territorio que ocupan, así como también con el factor humano, vale decir, con los ciudadanos que la habitan.

Uno de los requerimientos de la sostenibilidad de las ciudades es su transformación de lugares usualmente focos de contaminación, congestión e inseguridad, en ciudades saludables que redunden en una mejora de la calidad de vida de sus habitantes.

La Organización Mundial de la Salud (OMS), en su propuesta de proyecto Ciudades Saludables bajo un enfoque de salud, definió lo que es una ciudad saludable, en línea con el desarrollo urbano sustentable: “... [la ciudad saludable] es una que se encuentra involucrada en el proceso de crear, expandir y mejorar los ambientes físico y social y los recursos comunitarios, que permiten a las personas apoyarse mutuamente al desarrollar todas las funciones de la vida y desarrollar su máximo potencial. Una ciudad saludable en el futuro tendrá un ambiente físico limpio, seguro y de alta calidad; y operará dentro de su ecosistema. Las necesidades básicas humanas de los ciudadanos (comida, agua, habitación, seguridad y trabajo) serán satisfechas $[\ldots]$ La ciudad tendrá una economía vital, diversa, y sus ciudadanos tendrán un fuerte sentido de conectividad con su patrimonio biológico y cultural, así como con otros grupos e individuos dentro de la ciudad. El diseño físico y de gobierno de la ciudad será compatible con todas estas circunstancias y las apoyará..." (Devuyst, 2001: 28).

Dentro de esta perspectiva, se justifica el énfasis en el concepto sistémico de la ciudad, que supone el trabajo por espacios habitables, justos y equitativos; la necesidad de satisfacer las necesidades básicas de los ciudadanos presentes y futuros; la necesidad de una economía diversa y el desarrollo equilibrado con respeto al medio ambiente, entre otros.

Otra línea de interés para la construcción de una ciudad sostenible se desprende de la visión de las ciudades no sólo como espacios para la vida, como entes meramente consumidores, sino como fuentes de múltiples recursos, y cuyos ciudadanos, o al menos una parte de ellos, se dediquen a trabajar por la mejora de su entorno. En este sentido, la agricultura, una actividad que tradicionalmente se consideraba ajena a las actividades urbanas, se concibe ahora como una posibilidad cada vez mayor de integrarse a la ciudad.

Esta forma de agricultura que se lleva a cabo dentro de los límites o en zonas aledañas a las ciudades, se conoce como Agricultura Urbana y Periurbana (AU / AP) y constituye hoy una práctica internacional tanto del mundo desarrollado como de los países en vías de desarrollo.

La agricultura urbana es la práctica que se realiza en espacios urbanos dentro de la ciudad o en los alrededores, en zonas blandas (como 
traspatios, lotes) o en zonas duras (terrazas, patios) utilizando el potencial local que involucra la fuerza de trabajo, el área disponible, el agua de lluvia, los residuos sólidos, articulando conocimientos técnicos y saberes tradicionales con el fin de promover la sostenibilidad ambiental y generar productos alimenticios limpios para el autoconsumo y comercialización, fortaleciendo el tejido social.

El término "Agricultura Urbana y Periurbana" (AUP) fue propuesto en 1999 por la FAO con el objeto de referirse a un tipo de agricultura que se constituyó en el marco de la seguridad alimentaria en los países subdesarrollados, aunque también está en franca expansión en países desarrollados, pero con otros objetivos.

La Agricultura Urbana se vislumbra, por tanto, como un proceso que permite aportar a ciertos aspectos de la sostenibilidad de las ciudades, en la medida que:

- Se logran satisfacer en cierta medida las necesidades alimenticias y las necesidades de disposición de los residuos orgánicos; y se contribuye a la creación de mercados alternos, diversificando la economía.

- Al trabajar la tierra, se logra cierta sensibilidad por el medio natural. Esta sensibilidad puede derivar en la consideración por las generaciones presentes y futuras de los habitantes urbanos.

- Esta sensibilidad puede permitir el cuidado y preservación de ciertas características, prácticas y tradiciones culturales relacionadas con el ambiente. Es decir, puede lograrse un cambio de valores, instituciones y pautas sociales.

- Puede empezar a vislumbrarse la relación de todo con el todo; es decir, una conceptualización sistémica de las ciudades y los ciudadanos, y sus relaciones inmediatas y globales con otros ciudadanos, con otros seres humanos, con la naturaleza.

- Este reconocimiento de interdependencia puede llevar a fortalecer el apoyo mutuo entre ciudadanos para desarrollarse; empezando a crear una comunidad fuerte.

La Agricultura Urbana (AU), por tanto, puede ayudar a contribuir en mayor o menor medida a la construcción de una ciudad sostenible: por su contribución al ahorro e ingresos familiares; a la educación; a la provisión de espacios para la recreación y el contacto con la naturaleza; a los beneficios ambientales, como la conservación de áreas verdes, la infiltración de agua, el aumento de la biodiversidad en un ambiente construido, el aprovechamiento de los residuos orgánicos, las aguas residuales y el agua de lluvia; entre muchas otras contribuciones a la ciudad y sus ciudadanos.

En ciudades como Lima, con limitados antecedentes en su planeamiento, no se cuenta 
con informes científicos sobre la importancia, la función y los aportes de la naturaleza a la calidad del ambiente y a la vida humana en los ambientes urbanos consolidados. Por ello, es pertinente adelantar investigaciones que ayuden a conocer la mejor manera para hacer que la naturaleza se incorpore tanto al sistema de espacios públicos que conforman la estructura de la ciudad, como a aquellas áreas reconocidas por su zonificación como zonas de protección. Esto, porque especialmente en ellas se manifiesta una gran riqueza y diversidad de hábitats, caracterizados muchos de ellos por nuevas y muy diversas comunidades de plantas y animales, a menudo ajenas a la región, pero que se adaptan al microclima modificado que permiten las ciudades. Estos vestigios de comunidades de plantas nativas e introducidas, existentes en todos los rincones de las ciudades, requieren una catalogación y evaluación de sus funciones para reconocer su valor y proponer su manejo y conservación en pro del desarrollo sostenible de las ciudades.

Las áreas verdes, lugares que son hábitats potenciales para plantas y animales, tales como los fragmentos de bosques o prados, los corredores bióticos y demás conectores, deben integrarse a la planificación del territorio urbano, más si se tiene en cuenta que estos lugares proporcionan, además del enriquecimiento del paisaje, funciones ecológicas y de mejoramiento de la calidad de vida urbana, y oportunidades alternativas para experiencias recreativas y educativas.

\section{LA AGRICULTURA URBANA}

La FAO entiende la agricultura urbana (AU) como: "pequeñas superficies (por ejemplo, solares, huertos, márgenes, terrazas, recipientes) situadas dentro de una ciudad y destinadas a la producción de cultivos y la cría de ganado menor o vacas lecheras para el consumo propio o para la venta en mercados de la vecindad" (Méndez, et al. 2005: 56). El Centro Internacional de la Papa, PerúCIP, por su parte, concibe la AU como "las actividades de producción agrícola, procesamiento y distribución -dentro y alrededor de ciudades y pueblos- cuya motivación esencial es la generación de consumo e ingreso personales; las cuales compiten con otras actividades urbanas por recursos urbanos escasos de tierra, agua, energía y mano de obra (...) incluye actividades de pequeña y amplia escala en horticultura, ganadería, producción de cereales y leche, acuicultura y forestería" (Urban Harvest, CGIAR: 2006).

En 1996, en la cumbre mundial de la alimentación que organizó la FAO en Roma, se reconoció como prioritario el estudio de la agricultura urbana y periurbana, así como el mejoramiento de la eficiencia de los sistemas de abastecimiento y distribución de alimentos en las ciudades.

En el 2001, en Quito, se realizó el seminario internacional "La agricultura urbana en las ciudades 
del siglo XXI", donde se presentó gran cantidad de trabajos y se concluyó con la "Declaración de Quito", en la que se aboga por un desarrollo sostenible de las ciudades que incorpore a la agricultura urbana en la agenda política y en la planificación urbana.

Actualmente, la AU se ha constituido en una alternativa para enfrentar los problemas que se derivan del proceso creciente de urbanización que viven las ciudades y de los problemas de pobreza, inseguridad alimentaria y bajos niveles de calidad de vida que del anterior se derivan. Por este motivo, la literatura y las investigaciones realizadas respecto a este tema es bastante extensa, sobre todo, por la multiplicidad de experiencias de AU en el mundo, cada una de ellas con particularidades específicas (fundamentalmente porque depende del contexto en el que se desarrollan las estrategias). En suma, según el PNUD y la FAO, "se estima que alrededor del mundo 800 millones de personas se dedican a la Agricultura Urbana y desempeñan un papel importante en la alimentación de las ciudades de todo el mundo".

En esta medida, la AU, al integrarse en el proceso de la construcción de una ciudad sustentable, posibilita los componentes de este proceso lo cual no quiere decir, de ninguna forma, que la adopción de estrategias que promueven la $\mathrm{AU}$ no implique limitaciones y dificultades.

En primer lugar, la AU contribuye, en cierta forma, a mejorar la calidad del medio ambiente, aunque por sí sola no es la solución a los problemas medioambientales que presentan las ciudades: en muchas experiencias, las personas que hacen AU utilizan desechos orgánicos para la elaboración de abonos orgánicos para los cultivos, riegan los cultivos con aguas residuales, y reutilizan desechos para la construcción de espacios para cultivar (como el caso de las botellas de plástico). La AU también ha surgido como un instrumento para "mitigar el proceso de pérdida de biodiversidad, incluyendo el cultivo de plantas, cría de ganado o acuacultura en los asentamientos humanos" (Santandreu, Perazzoli, Dubbeling, 2002: 10).

Asimismo, la AU, dado el hecho que surge (por lo general) como una iniciativa individual, familiar y/o comunitaria, al institucionalizarse por medio de proyectos, programas, $y$ políticas, posibilita el establecimiento de espacios de participación para la discusión y la concertación respecto a la diversidad de experiencias de $\mathrm{AU}$, y a los alcances de ésta. Se considera que es necesario, al momento de formular las intervenciones gubernamentales, abrir los espacios de participación para que los agricultores urbanos tengan la posibilidad de dar a conocer sus experiencias y sus perspectivas respecto a la AU. Sólo así se garantiza la inclusión participativa de los actores involucrados -que fortalecen, por consiguiente, sus compromisos con el desarrollo del proceso-, la concertación de los diferentes 
intereses, y la aplicación más real de la AU y su consolidación como alternativa de construcción de ciudad sostenible. De igual forma, la AU fortalece las organizaciones existentes, potencia la creación de unas nuevas, e incluso posibilita la constitución de redes entre las mismas organizaciones.

La AU practicada actualmente en muchas ciudades del mundo, constituye un modelo de actividad que contribuye al logro de la Sostenibilidad Urbana en cuatro dimensiones: la ambiental, con la conservación de áreas verdes al interior de la ciudad y el aprovechamiento de residuos orgánicos y aguas residuales en el espacio de cultivo; la económica, con la creación de empleo y ahorro a nivel familiar; la social y la cultural, con la oportunidad de fomentar actividades y espacios de interacción e intercambio de conocimientos y tradiciones.

Los ciudadanos necesitan de un ambiente vivo donde crezca lo verde y sea parte de las ciudades. La agricultura urbana también puede ayudar a mantener esas áreas verdes. Obviamente, cada ciudad es diferente y podrá orientar la aplicación en su territorio específico.

En consecuencia, la AU pretende generar una producción alimentaria en un entorno urbano de manera auto gestionada en distintas escalas. En términos generales, la expresión AU ha sido aplicada para denominar la producción agrícola en huertos urbanos. Esta surge como potencial plataforma de desarrollo alternativo social, asumiendo el desafio de mejorar espacios degradados, ocuparlos y dar una puesta en valor de él y la potencialización entre la recuperación de los recursos del hábitat y la creación de actividades productivas agro-culturales, generando un encadenamiento operativo de la dimensión ecológica, económica y social(visión integral).

Mediante el cultivo de todo sitio disponible en el espacio abierto, incluso sobre los techos, los agricultores urbanos contribuyen a incrementar las áreas verdes de la ciudad, contribuyendo con su imagen urbana, reduciendo la contaminación, mejorando el suelo y la temperatura de una zona urbana.

Pero si bien la AU es una práctica con una ya larga historia internacional y regional, es muy nuevo su abordaje desde la perspectiva de la gestión urbana y territorial. Hoy comienzan a ser visualizadas con mayor claridad, tanto en la teoría como en la práctica, las múltiples formas en que la Agricultura Urbana interactúa con los diferentes elementos del desarrollo urbano, y empiezan a caminarse los primeros pasos para asumirla como parte integral de las estrategias de gestión urbana impulsadas a escala municipal.

En esta perspectiva, la AU puede ser un aporte a la construcción de una gobernabilidad democrática en las ciudades de los países en vías de desarrollo. La AU ofrece la oportunidad para que distintos grupos vulnerables -tradicionalmente excluidos de los sistemas políticos y económicos-se 
involucren en la vida socioeconómica de la ciudad, a través de la práctica de una actividad que tiene relación con sus conocimientos y prácticas, facilitando mecanismos de participación comunitaria en la gestión ambiental y el uso de la tierra. La AU re-valoriza la cultura tradicional y puede reforzar la identidad y responsabilidad individual y comunitaria de respeto al entorno.

\section{AGRICULTURA URBANA Y CONSERVACIÓN DE LAS ÁREAS VERDES EN LAS CIUDADES}

La recuperación de las áreas verdes es una prioridad de las ciudades. A través de una adecuada planificación y diseño del conjunto de áreas verdes de la ciudad es posible obtener una serie de beneficios que, siendo compatibles con la tradicional funcionalidad recreativa y embellecedora de estos espacios, contribuyan a una notable mejora de la calidad ambiental del medio urbano.

Los espacios verdes, principalmente a través de la vegetación arbórea, contribuyen a la reducción de la contaminación atmosférica, realizando esta función mediante la captación directa de compuestos presentes en el aire $(\mathrm{CO} 2, \mathrm{NO} 2, \mathrm{SO} 2, \mathrm{O} 3$, partículas, etc.), o bien de forma indirecta, moderando la temperatura urbana, lo cual contribuye a una disminución de las emisiones de gases contaminantes relacionadas con el consumo energético ligado a la refrigeración de los edificios.

Otra importante función del verde urbano es su papel en la moderación del clima urbano. Como se sabe, la presencia de la ciudad modifica profundamente el clima local, dando como resultado un microclima urbano artificial más cálido, determinado fundamentalmente por la geometría urbana, las propiedades térmicas de los materiales de construcción, el calor emitido por la actividad de los ciudadanos, y la distribución y abundancia de la vegetación. El papel de la vegetación en la moderación de la temperatura urbana se manifiesta a través de dos vías: por un lado, el efecto de sombra sobre las superficies que absorben la radiación solar, y, por otro, a través de la evapotranspiración.

Así mismo, destaca el papel que juegan los espacios verdes en relación con el mantenimiento de la biodiversidad urbana. La ciudad en sí puede ser entendida como un mosaico de hábitats; de hecho, la mayor diversidad de condiciones ambientales que se generan en la ciudad determina la existencia de una estructura de flora y fauna más diversificada inclusive que la de los entornos rurales. La biodiversidad urbana está ligada en gran medida a la presencia de espacios verdes en la ciudad que actúan como hábitat, refugio, fuente de alimento o medio de conexión con el entorno no urbanizado. Los objetivos de una planificación del verde urbano orientada a la conservación de la biodiversidad incluirian la provisión de la mayor diversidad posible de hábitats viables a largo plazo y con mínimas necesidades de mantenimiento; la búsqueda de compatibilidad entre la presencia 
de las distintas especies animales y vegetales; y la salud, seguridad y bienestar de los ciudadanos.

Ahora, ningún programa de áreas verdes estaría completo si no incorpora actividades de la agricultura urbana. La AU incluye actividades como la acuicultura, jardinería comercial, floricultura, huertos familiares, cultivo de plantas medicinales $y$ forraje, manejo de viveros, entre otros. Se estima que dentro de las ciudades estas actividades se pueden dar en más de la mitad de todos los usos "verdes" de los terrenos urbanos. Mediante el cultivo de todo sitio disponible en espacio abierto, incluso sobre los techos, los agricultores urbanos contribuyen a incrementar las áreas verdes de la ciudad, ayudando a reducir la contaminación, y a mejorar el microclima y la calidad del aire. Incluso el hecho de que una menor cantidad de alimentos tenga que ingresar a la ciudad en camiones contribuye a la sostenibilidad y tiene un impacto ambiental positivo. La AU ayuda a reducir la huella ecológica de una ciudad, aun cuando la ciudad continúa creciendo.

La AU tiene un impacto positivo en el enverdecimiento y limpieza de las ciudades, transformando los terrenos baldíos abandonados y, por lo general, usados como basurales informales o espacios abiertos, en zonas verdes, manteniéndolas como áreas libres de zonas residenciales, las cuales producen un efecto positivo sobre el microclima: sombra, temperatura, captura de $\mathrm{CO} 2$, etc.
Cuando se convierten estos espacios en "verdes", no sólo se resuelve un problema sanitario sino también una situación social, pasando los vecinos a disfrutar pasiva o activamente del nuevo espacio ganado. Tales actividades enriquecen la autoestima de los ciudadanos y estimula otras acciones para mejorar el nivel de vida de la comunidad.

El concepto de Agricultura Urbana es una herramienta para mejorar la calidad de vida de las familias. Tener un mini huerto en casa trae grandes beneficios y es relativamente fácil de lograr. El elemento principal es la disposición de crear en el hogar un espacio verde, por muy dificil que parezca.

Cultivar en casa no sólo es una alternativa sana para quienes prefieren lo natural. Los beneficios abarcan el área de alimentación, salud, consumo responsable, saneamiento ecológico, contribuyendo en la disminución de gases de efecto invernadero, el embellecimiento del jardín y del hogar, entre otros.

No se necesita tener jardin para poder cultivar vegetales o frutas, únicamente hay que tener en cuenta la necesidad que tienen las raíces de éstas para desarrollarse. Por ejemplo, cualquier hortaliza necesita al menos $40 \mathrm{~cm}$ de profundidad para crecer correctamente, ya sea en maceteros o en jardín.

En este contexto, los espacios para la AU dentro de un sistema de áreas verdes pueden ser integrados dentro del reuso de aguas, el reciclaje de basuras urbanas, y pueden ser 
particularmente importantes para mejorar la dieta y calidad de vida de la población de menores recursos y de todos los que deseen disfrutar del consumo de alimentos orgánicos.

Es entonces importante para el desarrollo urbano de las ciudades, tomar en cuenta actividades de la agricultura urbana como los huertos urbanos, así como los parques y jardines, que ofrecen la oportunidad para que los habitantes de las ciudades entren en contacto con la naturaleza. Además, presentan otras ventajas importantes: son indicadores de un cambio de valores, recuperan espacios urbanos abandonados o degradados, satisfacen parte de la demanda de productos ecológicos de bajo costo, fomentan las relaciones sociales $\mathrm{y}$ el intercambio de experiencias y productos, ya que se trata también de áreas de ocio al aire libre. Pueden también armonizar con los usos urbanos que se dan en las ciudades, aumentar los índices de área verde por habitante y, es más, generar un nuevo indicador de sostenibilidad de "área verde productiva" por habitante, acercándose cada vez más al ideal de las ciudades sostenibles.

\section{ESPACIOS PARA EL DESARROLLO DE LA AGRICULTURA URBANA}

El ejercicio de la agricultura dentro de las ciudades no es reciente, aunque desde finales de la década de 1980 se ha oído hablar cada vez más de la agricultura urbana (AU) en el mundo. Se ha desarrollado como fuente indispensable de alimentación para las personas con mayores carencias alimentarias e instrumento de reclamo para una alimentación más saludable y un ambiente menos contaminado, gracias a la generación de áreas verdes de tipo productivo que la $\mathrm{AU}$ produce. Antiguamente, se manifestó a través de la relación de la ciudad con los jardines urbanos y periurbanos en el transcurso de los siglos,

En cuanto a la zona de transición entre ciudad y campo, todavía sin urbanizar o parcialmente urbanizado, suele denominarse espacio periurbano. No es campo ni ciudad sino una zona de transición o interfase entre lo rural y lo urbano. Es, además, una zona de intensos conflictos de interés entre las actividades productivas primarias y la urbanización.

El periurbano supone un complejo territorial que expresa una situación de interfase entre dos tipos geográficos aparentemente bien diferenciados: el campo y la ciudad. Se trata de un territorio en consolidación, bastante inestable en cuanto a la constitución de redes sociales y de una gran heterogeneidad en los usos del suelo.

Estas áreas que rodean a las ciudades resultan de una importancia crítica para el mantenimiento de la calidad del aire y del agua del espacio urbano amanzanado y, lejos de ser protegidas, se convierten en un ambiente contaminado. Hay experiencias en las que estas áreas han sido motivo de recuperación al ser utilizadas para la práctica de la agricultura urbana y periurbana. 
Las expresiones: agricultura urbana, urban agriculture, agriculture urbaine o huertos urbanos, se refieren a superficies reducidas situadas en el perímetro urbano que se destinan al cultivo intensivo y la cría de pequeños animales domésticos, principalmente gallinas $\mathrm{u}$ otros similares; aunque también, solo que raramente, vacas lecheras. Esta producción se realiza principalmente en solares vacíos, patios y terrazas que se transforman en huertos comunitarios y familiares; y es practicada exclusivamente por personas que viven y trabajan en las ciudades.

Por otro lado, la agricultura periurbana tiene una connotación más amplia, y puede abarcar desde la mini agricultura intensiva y de subsistencia a la agricultura comercial realizada en el espacio periurbano. Las primeras tentativas de definirla o conceptualizarla proceden de la décadas de 1970 y están relacionadas con la teoría de la localización de Johann Heinrich von Thünen, desarrollada en la tercera década del siglo XIX y que estudia la relación y la distribución espacial de las actividades productivas alrededor de las ciudades.

En el área metropolitana de Lima ya se están abriendo los espacios para promover la práctica de la AU. Más de 30,000 mujeres y hombres de 16 distritos de Lima se beneficiarán con el programa de agricultura urbana "Mi Huerta", que pone en marcha el municipio limeño, como parte de sus politicas de generar áreas verdes para la conservación del medio ambiente y para contribuir a revertir la desnutrición, provocada por la pobreza extrema.

Este programa tiene como objetivo apoyar y fomentar la agricultura en la ciudad, contribuyendo a mejorar la nutrición familiar y los hábitos alimenticios a través del cultivo de vegetales, hierbas aromáticas y medicinales. Asimismo, promoverá el enverdecimiento de la ciudad con áreas verdes productivas.

De llevarse a cabo las prácticas de desarrollo de la AU para nuestra capital, tal vez de esta manera podría empezar a acercarse a lo que Richard Rogers, en su "Cities for a small planet", (2000), define como Ciudad Sostenible: una ciudad justa, bella, creativa, ecológica, que favorezca la sociabilidad; que sea compacta y policéntrica, además de diversa. Una ciudad, en fin, digna de vivir en ella. 


\section{FUENTES Y REFERENCIAS BIBLIOGRÁFICAS}

MOUGEOT, L.J.A. (2000). Urban Agriculture: definition, presence, potentials and risks.

CABANNES, Y.; MOUGEOT, L:J:A (1999). El estado de la Agricultura Urbana en América Latina y el Caribe.

ALBURQUERQUE, R. (1996). Agricultura Urbana, medio de generación de renta y transformación social.

www.cipotato.org/urban harvest/spanish/home.htm

www. Ruaf.org/urban agriculture

www. Idrc.ca/en_foco_ciudades/cultivando megaciudades 